Version de travail de :

Amuri Mpala-Lutebele, Maurice ; Halen, Pierre. « Italianité rhodiote et métissage africain dans Sang mêlé d'Albert Russo ", in : Des Italiens au Congo aux Italiens du Congo : aspects d'une glocalité. Sous la direction de Daniele Comberiati, Rosaria Iounes-Vona et Pierre Halen. Paris : L'Harmattan, coll. Mémoires lieux de savoir, 2020, 366 p. ; p.247-262.

\title{
ITALIANITÉ RHODIOTE ET MÉTISSAGE AFRICAIN DANS SANG MÊLÉ D'ALBERT RUSSO
}

\author{
Maurice AMURI Mpala-Lutebele ${ }^{1}$ \\ Université de Lubumbashi \\ Pierre HALEN \\ Université de Lorraine
}

Les relations de l'Italie avec l'Afrique centrale, particulièrement avec le Congo, passent également par les représentations littéraires. Si ces dernières sont susceptibles de s'inscrire dans les « débats internationaux suscités par l'État léopoldien et [...] exploitent les rêveries exotiques et d'autres aventures liées au cadre de la forêt primitive », comme l'indique l'argumentaire de ce colloque, l'œuvre romanesque d'Albert Russo s'inscrit quant à elle dans de tout autres débats, et notamment dans les questions relatives à la rencontre des cultures dans un contexte de globalisation. Le fait est qu'il s'agit d'une œuvre relativement récente, à divers égards post-coloniale, et qu'elle présente plusieurs aspects singuliers. Outre la dimension interculturelle déjà mentionnée, ne retenons ici que deux d'entre eux : d'abord, cette œuvre se ressent de l'expérience des Italiens provenant des îles de la mer Égée, particulièrement des communautés sépharades de l'île de Rhodes ; cette origine semble bien avoir constitué un fait inspirateur, et comme un cadre de pensée pour Albert Russo qui est lui-même un descendant direct de ce groupe social. Ensuite, cette œuvre prend à plusieurs reprises pour sujet et pour décor socio-historique l'établissement de ce groupe au Katanga, dans un contexte à divers égards étranger puisqu'il est à la fois congolais et francophone, et, selon les époques,

1 Voir : http://mukanda.univ-lorraine.fr/auteurs/maurice-amuri-mpala-lutebele 
colonial belge ou post-colonial. Dès lors, on conçoit que la question de l'Autre - qu'il s'agisse des différentes mémoires en concurrence ou des partenariats à négocier dans la vie urbaine - soit centrale dans les grands romans d'Albert Russo, notamment dans Sang mêlé en 1990, le roman auquel nous nous attacherons spécialement, mais aussi dans L'Ancêtre noire en 2003, et dans Exils africains en 2010.

« Né au [Congo-belge] de père italien et de mère anglaise, [Albert Russo] passe toute sa jeunesse sur le continent africain, entre l'ex-Congo belge, l'Afrique du Sud et le Burundi $»^{2}$. Toute son enfance et son adolescence, jusqu'à son départ pour l'université aux États-Unis, ont donc pour cadre l'Afrique centrale, du Sud-Katanga au Tanganyka ${ }^{3}$. S'il a aussi été, plus tard, un grand voyageur de par le monde, le fait est que les univers diégétiques de ses grands romans sont situés en Afrique, et notamment au Katanga, la province congolaise qui l'a vu naître en 1943 à Kamina, et puis grandir dans un contexte colonial finissant, encore structuré par des différences à outrance, mais qui vacille et s'ouvre à autre chose. À l'instar d'un Le Clézio qui spécule à propos de «l'Africain » qu'était son père, Albert Russo fonde lui aussi, consciemment ou inconsciemment, une grande partie de sa vision de l'humanité sur son expérience africaine, vécue comme une expérience de la diversité.

2 Russo (Albert), Sang mêlé ou ton fils Léopold [1990]. Paris : France-Loisirs, 1991, 264 p. ; prière d'insérer. Nos citations renverront à l'édition originale: Sang mêlé ou ton fils Léopold. [Préface de Michel Fabre]. Boulogne : Éditions du Griot, 1990, 258 p. ; en abrégé : $S M$. Le roman a été traduit en italien : Sangue misto. Traduzione di Marco Dorner. Roma : Coniglio editore, 2008, 208 p. ; Idem. Postfazione di Michel Fabre. Roma : Elliott, coll. Scatti, 2016, 178 p.

3 « Renseignements sur mon père italien, Moïse Russo, et ma mère britannique, Sarah : mon père, né Italien à Rhodes (sous occupation italienne après la chute de l'empire ottoman) en 1910, est arrivé au Congo à 16 ans. Il a travaillé pour Amato Frères à Kamina, à Luluabourg, à Jadotville, à Élisabethville, puis s'est associé en 1950 avec son frère Jacques et Léon Hasson, dit le Turc, avec magasin de gros, boutique pour dames, Diana, et usine de sousvêtements dans le quartier industriel de Lubumbashi (ex-Élisabethville). Après des démêlés avec ses associés, ma famille s'est transférée à Bujumbura (Burundi) en 1955, où elle a repris une affaire mal en point, que, à force de travail, elle a fait prospérer. Ce qui est devenu "M.A. Russo". Ma mère et mes sœurs ont émigré en Italie en 1959, tandis que moi, je suis resté à Bujumbura pour terminer ma Rhétorique (Bac belge), puis je suis allé faire mes études universitaires à New York. Mon père est resté encore de nombreuses années à Bujumbura jusque dans les années 1975-1978. Ma mère, Sarah, a grandi en Rhodésie/Zimbabwe, a fait un voyage à Élisabethville/Lubumbashi en 1941, y a rencontré mon père et ils se sont mariés à É'Ville, en 1942. Moi, je suis né à Kamina en 1943, ma sœur Rachel, à Jadotville en 1946, et ma sœur Irène à É'Ville/Lushi en 1949. Je suis donc né Italien, puis mon père nous a tous naturalisés belges. Nous recevions les personnes de toutes races et religions à la maison. Et moi, pendant six ans, j'ai fréquenté à Bujumbura l'Athénée Royal Interracial, avec dans ma classe des Blancs, des Tutsi, des Hutu, des Congolais, des Pakistanais, des Arabes (en minorité, il est vrai, mais c'était une "révolution"). Ces éléments et cette situation à la fois familiale et scolaire m'ont ouvert au monde et à l'acceptation de toutes les différences à une époque où la ségrégation raciale était un fait ». (Correspondance d'Albert Russo du 15 octobre 2014). 
C'est elle qui lui inspire un discours à propos de la complémentarité des cultures et de leur enrichissement réciproque : toutes lui parissent en effet nécessaires à l'accomplissement d' " une humanité pas tout à fait déchue » (comme il l'écrit dans une de ses premières publications, significativement intitulée Éclats de malachite ${ }^{4}$ ). D'où la poétique et en même temps l'éthique du métissage qui seront, vingt ans plus tard, au cœur du roman Sang mêlé, comme nous allons le voir. Avant cela, ajoutons que le mélange est, d'une manière générale, la figure essentielle de l'œuvre, ne serait-ce que parce que celle-ci est écrite en deux langues (le français et l'anglais, l'auteur s'autotraduisant), mais aussi parce qu'elle touche à de très nombreux genres et supports, de la poésie à la photographie en passant par le roman pour la jeunesse et la chronique, et même le cinéma ${ }^{5}$.

\section{Trois voix pour mettre en situation les différences}

Sang mêlé est une fiction: on y raconte l'histoire de Harry Wilson, un Américain de la banlieue de Baltimore, qui immigre au Congo belge vers la fin des années trente à la suite d'une grave mésentente avec son père : «cet homme a gâché mon existence » $(S M$, p. 145), écrit-il. S'il arrive au Congo, c'est qu'il espère y être rejoint par son amant, qui a des projets missionnaires, mais celui-ci, mobilisé, meurt sur le front en Normandie. Installé à Élisabethville, aujourd'hui Lubumbashi, où il se consacre à des activités commerciales privées, Harry Wilson vit une migrance marquée par divers rapports avec une nouvelle société, de nouvelles langues et de nouveaux modes de vie: en somme, une interculturalité, et celle-ci sera racontée, après qu'un narrateur à la troisième personne aura introduit le roman, par plusieurs voix.

D'abord la voix de Wilson lui-même : « comment pourrais-je oublier ma première semaine à Élisabethville ? » (SM, p. 147), s'exclame-t-il. Se révèlent alors, tour à tour, ses contacts avec la société congolaise : avec Tshikapa, le premier jeune Congolais qui est à son service ; avec Mama Malkia, la gouvernante congolaise qui tient de main de maître sa maison ; avec Léopold Kitoko Wilson, le petit mulâtre qu'il adopte ; ses relations avec la société européenne aussi, notamment avec Jennie, à la fois sa conseillère et son employée ; et surtout avec ses amants successifs, puisque Harry Wilson a une liaison passionnée avec un Grec, Giorgos, mais aussi une relation plus éphémère avec un jeune Commissaire belge, Éric, et une «passade » avec un pilote rhodésien, Craig. La problématique de l'homosexualité dans une société peu tolérante introduit d'une autre façon celle du respect des différences.

Ensuite, c'est la voix de Mama Malkia, une veuve qui était

entrée au service de Harry Wilson peu après que l'Américain se fut établi à Élisabethville. Mama Malkia méritait bien son nom qui, en kiswahili, signifie « reine mère ».

4 Russo (A.), Éclats de malachite. Bruxelles : Pierre de Meyère, 1971, 171 p. ; p. 5.

5 Pour une bibliographie, consulter : http://mukanda.univ-lorraine.fr/ - c. 07.10.2019. 
[...] Mama Malkia avait un sens inné de l'organisation et m'sieur Harry n'hésitait pas à la consulter. Pour les tâches de la maison, c'est elle qui commandait $(S M$, p. 22).

Son service auprès du Blanc Wilson est l'occasion d'un dialogue parfois tumultueux, surtout lorsqu'il est question des liaisons de Harry et de l'éducation de Léopold Kitoko dans ce contexte. Ce dialogue a lieu en même temps entre les cultures occidentales et africaines, puisque Mama Malkia se fait l'écho de ses concitoyens congolais. Ceux-ci, en effet,

émirent $[. .$.$] quelques réserves sur l'attitude de m'sieur Harry ; pas tant sur l'adoption$ de Léo - l'humanité n'était après tout qu'une grande famille -, que sur l'homosexualité du Blanc. [...] Quelle valeur un homme pouvait-il avoir s'il abandonnait le premier de ses devoirs qui était de procréer? (SM, p. 239).

La troisième voix, enfin, celle de Léopold Kitoko Wilson, raconte la perplexité d'un jeune homme "pris entre deux mondes », en quête de son identité : «oui, papa, avoue-t-il, j'aurais souhaité être uniquement d'une race, et non un café-au-lait. Je me sens, corps et âme, si inadéquat, si inachevé » (SM, p. 127).

Dans l'ensemble, « chacune de ces voix nous parle de la difficulté à vivre sa différence », comme on peut le lire dans le prière d'insérer. Trois voix, trois consciences narratives pour une même réalité, un même drame. Trois voix qui, finalement, deviennent complémentaires pour faire voir une issue : un processus d'acculturation, qui conduit à valoriser le symbole du métissage : le « sang mêlé ». Martine Pretceille nous a rappelé que « les cultures comme les sociétés ne sont pas homogènes » et que « la pluralité et la diversité sont au cœur même de la vie $»^{6}$. Mais les univers sociaux concernés par l'immigration sont ceux où s'affichent le plus, et parfois s'affrontent, mais parfois aussi s'effacent finalement, les différences, ou du moins leur potentiel de division. Que dire alors d'une immigration vécue dans un contexte de colonisation lui-même marqué par beaucoup de stéréotypes et de catégorisations diverses concernant les uns et les autres?

Dans le roman, la première mise en situation des différences concerne naturellement le premier contact de Wilson avec son nouveau milieu physique où il constate que

certaines agglomérations se résumaient à quelques cabanes d'onduline et à une demi-
douzaine de huttes éparpillées. On avait l'impression de se trouver dans une autre gala-
xie. J'étais à la fois effrayé et fasciné par tant de démesure, et je me demandais si cela
n'avait pas été une folie de quitter Baltimore $(S M$, p. 147).

La mise en situation des différences se construit davantage dans les rapports entre colonisateur et colonisé, notamment à travers le «patron » Wilson et la « domestique » Mama Malkia, qui entend bien n'appeler Hary que « M'sieur

6 Pretceille (Martine A.), L'Éducation interculturelle. $4^{\mathrm{e}}$ éd. mise à jour. Paris : PUF, coll. Que sais-je ?, n³487, 127 p. ; p. 22. 
Wilson ». L'attitude de ce dernier, dans ces relations, n'est pas bien vue par d'autres Blancs. En effet, révèle-t-il,

les Européens de mes relations furent plongés dans la perplexité, et certains ne purent cacher leur désapprobation lorsqu'ils apprirent que, sur mon initiative, Mama Malkia avait quitté la cité indigène pour venir s'installer chez moi. Cela, tout simplement, ne se faisait pas (SM, p. 164).

Qu'une servante indigène s'asseye à leur côté et partage leur repas, cela pouvait à la rigueur se concevoir dans un village de brousse ou dans des fermes isolées, mais pas à Élisabethville! (SM, p. 46).

Comment Wilson pouvait-il se le permettre avec cette servante indigène ! Wilson se retrouve dans une autre situation de différence quand il décide d'adopter un orphelin, décision qui paraît insolite à la fois aux yeux des Blancs et des Noirs :

- Ainsi, vous voulez adopter un petit mulâtre ? répéta Janine sur plusieurs tons.

C'était une affaire trop grave pour qu'on s'y engageât à la hâte. Nous en parlâmes très longuement avant de parvenir à une conclusion. Je dis «nous » car sans la compréhension et le soutien indéfectible de Janine, je ne me serais jamais risqué à prendre une décision d'une telle ampleur. Quant à Mama Malkia, elle crut, lorsque je lui révélai mon projet, que j'avais « perdu la boule » $(S M$, p. 164).

L'intolérance à l'égard de la différence provient cette fois-là des deux côtés. Elle se prolonge par le rejet que le jeune mulâtre Léopold Kitoko Wilson et son ami Ishaya, Juif sépharade, subissent à l'école, de la part de leurs camarades blancs, à cause respectivement de leur couleur de peau (pour Léo qui pâtit aussi d'un double statut de bâtard et d'orphelin) et de leur religion (pour Ishaya). Rapportant une de ses altercations avec un camarade de classe, Léo raconte : «d'après lui, il est même plus que certain que j'irai droit en enfer, parce que je n'ai pas de vrai père ni de vraie mère et que je suis... à moitié noir » $(S M$, p. 135). Ishaya rapporte une anecdote semblable : « il m'a demandé pour quoi je n'allais pas à l'église. Je lui ai expliqué que j'étais juif et que par conséquent je ne pouvais prier qu'à la synagogue » $(S M$, p. 79).

L'homosexualité de Wilson est également un motif de confrontation des différences dans la mesure où non seulement elle provoque une certaine inhibition identitaire chez Wilson lui-même, mais elle est aussi «cause de son exclusion du cercle des colons » (1991, prière d'insérer) tout en apparaissant comme une pathologie aux yeux des Noirs. Wilson tient donc à cacher ses « tendances sexuelles », comme il les appelle, et il ne souhaite pas que son fils adoptif Léopold soit homosexuel :

Il a néanmoins le droit de savoir pourquoi je ne me conduis pas comme la plupart des autres hommes. Mon intelligent Léo, il n'est pas dupe. Mais je ne voudrais pas qu'il soit comme moi. Cela le... détruirait (SM, p. 145).

Par ailleurs, se confiant aux membres de sa famille élargie, Mama Malkia

leur raconta comment elle en était venue à travailler pour m'sieur Harry, le muzungu américain. Elle ne leur cacha pas le fait qu'il ne couchait pas avec les femmes. Cette 
révélation lui valut des regards désapprobateurs et déclencha les yo ! yo! de l'assistance, car le bwana était alors sûrement possédé (SM, p. 185).

Toutes ces mises en situation des différences montrent suffisamment que « les cultures comme les sociétés ne sont pas homogènes [et que] la pluralité et la diversité sont au cœur même de la vie ${ }^{7}$. Mais alors, comment gérer cette diversité, cette pluralité ? À l'intolérance à l'égard des différences, Albert Russo oppose une poétique du métissage, et sa vision d'une humanisation toujours plus grande des sociétés.

\section{Poétique du métissage}

Dans Exils africains ${ }^{8}$, Albert Russo expose clairement sa conception du métissage. Quelle attitude adopter, en effet, face au conflit que peut engendrer la cohabitation des différences ? Faut-il renier un côté pour embrasser l'autre exclusivement? Ou alors se détacher des deux, et ainsi se libérer de toutes les chaînes du passé afin de se forger une personnalité nouvelle ? (SM, p. 178) Comme le montre ce roman ultérieur ${ }^{9}$, Albert Russo choisit la seconde voie, celle d'une acculturation entendue ici non pas comme « assimilation », mais comme «ensemble des phénomènes qui résultent de ce que des groupes d'individus de cultures différentes entrent en contact, continu et direct, avec les changements qui surviennent dans les patrons culturels originaux de l'un ou des deux groupes ${ }^{10}$. Cela conduit à minimiser les différences culturelles et, en même temps, à souligner la complémentarité entre les groupes. "Seule cette démarche permet de rompre les logiques de violence au sein d'une même société ou entre deux sociétés que l'on tente de poser en rivales ${ }^{11}$.

Ce processus d'acculturation est représenté dans Sang mêlé par des procédés romanesques caractéristiques : une narration basée sur une alternance de tension et de détente, une polyphonie singulière et un mélange de genres significatif.

\section{Une alternance de tension et de détente}

Chaque mise en situation apparait comme un temps fort, un moment de tension du fait de l'affrontement des différences. Albert Russo fait suivre

7 Pretceille (M.A.), L'Éducation interculturelle, op. cit., p. 22.

8 Russo (A.), Exils africains. Et il y eut David-Kanza. Présentation de Pierre Halen. Paris : Ginkgo éditeur, 2010, $195 \mathrm{p}$.

9 Cf. Amuri M.-L. (Maurice), « Rencontre des cultures et quête d'identité : démystification du discours sur l'Autre dans Exils africains d'Albert Russo », in : QuAGHEBEUR (Marc), dir. ; LECLERCQ Nicole), collab., Sagesse et résistance dans les littératures francophones. Bruxelles : P.I.E.-Peter Lang, coll. Documents pour 1'Histoire des Francophonies, n ${ }^{\circ}$, 631 p. ; p. 357-372.

10 J. Redfield et alii, cités in : PretCEILle (M.A.), L'Éducation interculturelle, op. cit., p. 13.

11 Blu (D.), «Introduction », in : Albert (Odile) ; FlÉCHEUX (Laurence), dir., Se former à l'interculturel : expériences et propositions. Paris : Éd. Charles Léopold Mayer, coll. Dossier pour un débat, 2000, 140 p. ; p. 9. 
chacun de ces temps forts d'une détente, d'un compromis, d'une sorte de réconciliation vécue par de nouvelles identités qui relativisent les différences sans les effacer. Cette dédramatisation permet de réunir les hommes de cultures différentes et chaque tension est dès lors neutralisée par une forme de réunion.

Ainsi, l'Afrique, d'abord représentée par des stéréotypes tels que des lions qui se promènent dans la rue, des serpents et des scorpions dans tous les coins, etc., se révèle ensuite une sorte de paradis à l'arrivée de Wilson : "Élisabethville fut une agréable surprise, s'exclame-t-il. Je découvris une ville coquette avec ses maisons aux vérandas enlacées de bougainvillées écarlates ou orangées, ses avenues bordées de flamboyants et de jacarandas » (SM, p. 147). Plus tard, quand sa sœur Clara, restée à Baltimore, « vit des photos récentes d'Élisabethville, elle n'en revint pas. Il existait donc en pleine Afrique, aux abords de la jungle, des villes aux avenues asphaltées, aux grands bâtiments modernes, avec des jardins à la française, des cinémas et même des théâtres ! ( $S M$, p. 94). Ses exclamations révèlent finalement que la connaissance de l'Autre détruit les préjugés ; comme on va le voir, elle conduit aussi à la connaissance de soi-même, en permettant donc une nouvelle identité.

Les rapports entre colons et colonisés sont, quant à eux, figurés de manière paradoxale (au sens littéral du mot) par les deux personnages du bienveillant Wilson et de Mama Malkia «plus tout à fait africaine, mais noire aux yeux de tous » (1991, prière d'insérer). En effet, « les rapports unissant Mama Malkia et Harry Wilson étaient inhabituels à la colonie. Entre eux s'étaient tissés des liens d'authentique tendresse, voire de connivence » $(S M$, p. 25). Une connivence qui se réalise au-delà des désaccords qui demeurent :

non que tout allât sans accrochages entre Mama Malkia et moi. Je la trouvais autoritaire, ses manières étaient un peu rudes, et elle fourrait son nez partout. Elle m'appelait «m'sieur » au lieu de «bwana » et elle avait eu le toupet de me dire que je n'étais pas un homme à femmes (SM, p. 163).

La mise en situation d'un patron quelque peu soumis et d'une servante autoritaire débouche plutôt ici sur un compromis, sur une sincère collaboration de deux sensibilités complémentaires pour fonder une communauté unie, une « famille » où Mama Malkia joue pleinement son rôle de «mère ». Wilson le reconnaît quand il se rassure au sujet de l'épanouissement de son fils adoptif : « après tout, il a Mama Malkia, et c'est une mère irréprochable » (SM, p. 144).

Que Wilson et Mama Malkia transcendent en quelque sorte leur statut respectif interpelle forcément leurs milieux. Albert Russo explique l'évolution du premier en évoquant l'attitude de Janine, une Blanche, envers les enfants noirs :

son amour des enfants était si authentique qu'il ignorait les barrières raciales. Ainsi elle arrêtait une Négresse dans la rue avec un bébé au dos et, lui caressant la joue, elle disait : "l'adorable poupou" [...]. L'attitude de Janine à l'égard des enfants noirs déclencha en moi un élan dont l'issue fut irréversible (SM, p. 161-162). 
« Irréversible », reconnait Wilson, dans la mesure où sa « relation avec l'élément primordial du continent noir, [avec] son peuple » $(S M$, p. 183), demeurée jusque-là latente, est devenue en lui une préoccupation consciente :

les Mama Malkia de ce pays, [déclare Wilson,] pourraient nous donner à nous, diables d'étrangers, plus d'une leçon. Car eux aussi nous voient comme de drôles d'oiseaux se conduisant de la façon la plus extravagante. [...] Le jour où, forts de leurs traditions, ils auront aussi maîtrisé le sens de nos comportements, il nous faudra veiller à compter avec eux (SM, p. 183-184).

$\mathrm{Au}$ lieu de voir les Autres, de manière exotique, comme de «drôles d'oiseaux », il faut donc « compter avec eux » : cela paraît limpide. Le qualificatif d' "irréversible » a aussi un sens tout particulier dans la mesure où Wilson adopte Léo en dépit du regard discriminatoire des uns et des autres. Il ne regrette pas ce choix qui donne plutôt un sens à sa vie :

ai-je été trop égoïste, orgueilleux ou simplement naïf en pensant que, par l'adoption de Léo, je pourrais changer quelque chose à la société ? / J'ai probablement été tout cela. Mais personne ne pourra me faire regretter ce choix. Oui, j'ai pris un risque immense et il me faudra beaucoup de courage. C'est parfois effrayant, mais Léo est tout ce pour quoi je me bats (SM, p. 144).

Wilson se réalise ainsi dans l'Autre : « cet enfant figure déjà à mes yeux un autre moi-même; non pas un double, mais un être que je m'appliquerai à rendre plus achevé, plus parfait» $(S M$, p. 165). Non seulement l'adoption traduit ici une vocation de paternité sans frontière raciale, mais elle exprime surtout cette capacité humaine d'aller au-delà de toute différence pour s'épanouir avec l'Autre, en l'Autre ; il est à noter cependant que celui-ci est moins considéré comme tel que comme un "être humain», donc un semblable : «père novice, je viens à peine de découvrir ma nouvelle vocation, celle de donner à Léo tous les moyens qu'il faut à un être humain pour atteindre le bonheur. Tâche difficile mais ô combien passionnante !» (SM, p. 171).

La neutralisation de la différence vécue par le petit mulâtre dans l'école des enfants blancs se retrouve, comme en miroir, dans l'heureuse expérience de Piet, un enfant blanc inscrit dans l'école des Noirs, et dont le prénom connote une identité néerlandophone. Piet

fréquentait l'école d'une mission catholique voisine dont il était le seul élève européen. Ses parents ne voulaient pas l'envoyer à la ville en internat, arguant que le seul moyen pour [lui] de ne pas se sentir exclu parmi ses camarades congolais était d'apprendre leur idiome (SM, p. 108).

Encore une fois, il est question ici de combattre l'exclusion par l'intégration : «Piet, avec l'accord de ses parents, avait délaissé une part de son héritage flamand pour vivre à la congolaise » $(S M$, p. 112), la meilleure manière de devenir un « sang mêlé » culturellement. Piet apparaît alors comme un autre symbole du métissage culturel. Cependant, parce qu'il pratique dès lors couramment le kiswahili avec Ishayah, ceci installe une barrière entre Léo et les deux «Congolais blancs » qui le laissent à l'écart et se sont, en quelque 
sorte, davantage enracinés localement que lui : on peut tout intégrer, il y a des choix à faire qui sont aussi des séparations même s'ils sont des mélanges.

La différence que crée l'homosexualité de Wilson apporte, quant à elle, plus de questions que de réponses. Elle n'est pas le résultat d'une décision volontaire comme celle de l'adoption, mais elle demande tout de même aussi une sorte de conversion puisque l'individu doit se découvrir et s'accepter luimême. Parlant de ce qu'il ressent envers son premier amant John, Wilson confesse :

personne jusqu'ici n'avait occupé dans mon cœur une aussi grande place. Et c'était là, dans cette chambre, en cet instant, que cela me fut révélé. La lutte contre mes instincts, cette épreuve d'endurance, valait bien la peine d'être sacrifiée. [...] Nous échangeâmes un autre baiser et le soleil nous surprit dans les bras l'un dans l'autre. Les choses allèrent rapidement. Que nos destins fussent désormais liés, était une évidence (SM, p. 177).

Cette révélation semble naturelle, et pourtant Wilson ne donne pas l'impression d'être naturel dans l'accomplissement de ce « destin », surtout quand il ne veut pas que son fils adoptif soit comme lui. Cela le détruirait, dit-il. Mais «pourquoi en serait-il détruit?» $(S M$, p. 145), s'interroge-t-il par la suite. Dans le roman, la question demeure posée ${ }^{12}$.

Si le principe de l'acceptation des réalités s'impose sans trop de difficultés dans l'enthousiasme de l'amour qui est comme béni par le soleil, d'autres interrogations concrètes sont également soulevées, notamment à propos du devoir de procréation, à propos de conviction religieuse, etc. La neutralisation de la différence est souhaitée, mais il reste, dans la société, une intolérance comme le montre ce propos du petit mulâtre, s'adressant à son père :

quelqu'un, je ne sais plus qui, t'a traité de pédéraste. Cela m'a bouleversé, et j'ai
cherché le mot dans le dictionnaire. Mais pour tout l'or du monde, je ne voudrais pas
d'autre papa, mon papa... Certains croient que tu es né... pour ainsi dire handicapé,
mais tu ne leur prêtes pas attention. Tu estimes que c'est une question de conscience, si
intime, que, même moi, j'aurai à prendre une décision sur ce point. Tu affirmes n'avoir
pas eu le choix. [...] Papa, cessons ce discours, mon cœur va éclater (SM, p. 221-222).

La neutralisation de la différence se cherche encore, semble dire Albert Russo par ces questions restées en suspens, ce tourment intérieur. C'est sans doute qu'il ne faut pas la confondre avec une conciliation totale, mais y voir plutôt une dynamique.

À chaque temps fort, donc, sa détente, à chaque conflit des différences sa neutralisation, réalisée ou en cours de réalisation. Albert Russo fait ainsi alterner ces deux temps pour suggérer diverses formes de réconciliation, diverses formes d'acceptation de l'Autre. Cette altérité en quelque sorte active, dont nous avons vu qu'elle s'éclairait d'être à la fois subordonnée et éclairée par

12 L'explication anecdotique est peut-être à trouver dans la souffrance avec laquelle Harry vit, non pas son homosexualité, mais sa passion pour et avec Giorgios, ce dernier se montrant à l'occasion jaloux et violent, ce qui complique inévitablement la vie familiale 
un principe d'humanité commune, s'entend également dans la diversité des voix du récit.

\section{Une polyphonie singulière}

Roman à trois voix (en plus de celle du narrateur), Sang mêlé aborde la question de la différence telle qu'elle est ressentie par un Blanc, un Métis et une Noire. Trois narrateurs impliqués dans l'histoire qu'ils racontent, apportant chacun son regard sur la même réalité : la difficulté à vivre la différence. Albert Russo se sert de ces trois consciences narratives pour filtrer la même vision, sa vision : l'hybridation culturelle féconde. En effet, au lieu de se rejeter, ces trois différences communient au nom du principe commun de l'acceptation des différences. Blanc, Wilson accepte d'adopter un mulâtre pour qu'il devienne un autre soi-même; libéral, il accepte l'autoritarisme de Mama Malkia, nécessaire à la construction d'une «famille » unie. Pour tout l'or du monde, Léo ne voudrait pas d'un autre papa, bien que Wilson soit homosexuel. Mama Malkia, quant à elle, malgré des regards désapprobateurs des siens, accepte de «cheminer» avec Wilson dans l'adoption de Léopold. Wilson résume bien cette hybridation culturelle féconde quand il dit : « Mama Malkia est, avec moi, le seul être qui puisse lire dans le cœur de Léo. Mais nous venons, elle et moi, de mondes si différents. [...] C'est moi qui ai adopté Léo, mais elle en est devenue la vraie mère, une mère tigresse » $(S M$, p. 146). Ni les différences à valeur sociale (groupe d'origine, profession, genre sexuel, couleur de peau) ni les différences de caractère n'empêchent donc la construction d'une symbiose (toujours incomplète), ici d'une famille.

En fin de compte, les trois histoires se fondent en une seule, chaque voix se muant en l'autre avec une nouvelle identité : Wilson, Léopold et Mama Malkia ne sont plus uniquement un Blanc, un Mulâtre et une Noire, mais un Blanc, un Métis et une Noire privilégiant ce qui leur est fondamental et prenant chez l'autre ce qui est positif et bénéfique à l'épanouissement de tous. Wilson illustre la mue de ces trois voix en une seule quand il ne sait plus distinguer la sienne de celle de Léopold :

mais il m'advenait quelque chose d'étrange tandis que je parlais. On eût dit que je traversais un rêve les yeux grands ouverts. Le silence de Léo résonnait de mes mots. Ce n'était plus ma voix que j'entendais, mais la sienne. L'écho semblait reproduire un monologue inversé, ponctué des rumeurs feutrées qui nous cernaient (SM, p. 220 ; voir aussi p. 127).

On est donc passé de la perception des discours de division, diffusés dans la société par des rumeurs stigmatisant et creusant les différences, à l'adoption de préoccupations communes, mais sans préjudice pour des différences désormais vécues d'abord comme une polyphonie, puis comme symphonie comme le suggère cette dernière citation. 


\section{Mélange des genres littéraires, accueil des langues}

Le mixage est tellement devenu le fondement de la vision d'Albert Russo que son écriture romanesque n'échappe pas au mélange des genres, chacun d'eux apportant une touche particulière à l'ensemble. Alors que toutes les ressources de la prose romanesque de la tradition réaliste sont sollicitées pour mettre en situation les différences sur les plans social, culturel, sexuel, religieux, linguistiques, etc., le roman s'ouvre sur un poème évoquant le jaillissement du métissage. L'expression «sang mêlé » en est le refrain :

[...] // sang mêlé // C'est lui qui gicle dans vos veines / Globules rouges, globules blancs ; / De race il n'y a point, / Ni de ces couleurs qu'inventent les esprits aveugles / Car avant, bien avant les théories darwiniennes / En dépit des calamités historiques / De ces maladies collectives et endémiques // LE SANG déjà avait fait son choix / Du rouge vous vous lassez ? / Pauvres philosopheux, tristes leucémiques / Prêtez donc l'oreille au corps, à sa musique / Ces accords intérieurs, heurtés ou merveilleux / Mais où rien jamais ne meurt // sang mêlé ( $S M$, p. 15).

Le sang (sans majuscule à l'initiale : c'est une réalité commune), a donc « déjà fait son choix / du rouge », ce qui renvoie toutes les séparations de couleur au statut d'inventions par des « esprits aveugles ». Mais aussi, on l'aura noté, c'est la musique autant que le corps qui deviennent les espaces où cette unité, ce « commun » du sang se manifeste par des « accords intérieurs ».

Corps et musique sont des notions qui nous entraînent presque naturellement vers la question des langues, si l'on veut bien considérer que chaque langue est comme une musique du corps. L'intégration de Wilson à son nouveau milieu passe dès le début par la connaissance de la langue de l'Autre ; la suite de son aventure congolaise en sera bien entendu facilitée. A. Russo, à l'instar de tant d'écrivains africains qui utilisent une langue européenne, adopte lui aussi une forme de bilinguisme, mais dans son écriture, puisque le kiswahili abonde dans le texte et fait, selon les éditions, l'objet de notes ou d'un lexique en fin de volume.

Ajoutons que le genre épistolaire est également mis à profit, surtout pour construire et perpétuer les nouvelles identités : sous forme d'épilogue du récit, les lettres que Wilson et Mama Malkia adressent ainsi à Léo, parti en Amérique pour ses études, affichent nettement cette intimité que les lettres savent rendre. L'un signe : «ton père qui t'aime, Harry »; l'autre : «celle de qui ni la foudre ni les montagnes ne pourront te séparer » $(S M$, p. 255, 258).

De l'œuvre romanesque d'Albert Russo, le mélange est donc un aspect essentiel, qu'il s'agisse des langues, des genres littéraires, des expériences artistiques, des groupes humains, des générations et des lieux, ou des genres au sens sexuel : tous ces traits d'écriture construisent, en somme, une poétique du métissage qui est de toute évidence concertée. 


\section{Et l'Italie dans tout ça ?}

De l'Italie, il n'a guère été question jusqu'ici, sinon pour signaler que l'auteur était issu de ces familles rhodiotes sépharades, au départ italiennes, que les conditions matérielles difficiles, puis la menace que représentaient le fascisme et son allié nazi avaient poussées vers un nouvel exil durant l'entredeux-guerres, et cette fois vers des terres non italiennes. Harry Wilson n'est d'ailleurs pas Italien, peut-être parce que l'Italie restait associée à cette mémoire douloureuse, mais cette hypothèse, on le verra, s'avère fragile. Pourquoi alors avoir choisi un États-unien ? Une partie de la réponse tient à la logique narrative : du moment que l'auteur voulait envoyer son personnage (comme dans son propre cas) faire des études supérieures en Amérique, faire de son père adoptif un natif de Baltimore dont la sœur serait prête à accueillir son neveu était une manière de donner de la cohérence à son récit. Cette explication par la biographie a ceci d'intéressant qu'elle suggère que Léo Kitoko est une auto-image au moins partielle de l'auteur, mais toute justification par la biographie ne saurait être que partielle et précaire. Reste donc à expliquer autrement le choix d'une nationalité américaine.

Cette américanité est sans doute, pour une part, à mettre en rapport avec une identité katangaise, telle qu'elle s'est imaginée, à partir de la Seconde Guerre mondiale et surtout après 1945, comme «carrefour du monde ${ }^{13}$, détachable au besoin du monde ancien que semblait de plus en plus être la Métropole. La province, où la présence anglaise avait été initialement très importante, s'était certes autant que possible belgifiée avec les grandes sociétés dites de 1906 (UMHK, CSK, BCK) et le régime colonial proprement dit, mais, par la force des choses, les relations avec l'Afrique australe anglophone n'ont jamais cessé et la Seconde Guerre mondiale les a encore renforcées avec la présence régulière de «troupes britanniques et sud-africaines » en transit, qu'évoque d'ailleurs le roman (SM, p. 151). Sang mêlé parle à une autre reprise de ces troupes alliées, lorsque Harry Wilson a un amant nommé Craig Butler, un Rhodésien, type même de l'« Africain blanc», complètement affranchi de l'Europe métropolitaine, mais pas pour autant rapproché des populations autochtones. Or, cette liaison ne dure pas, et l'explication qui en est donnée par Harry à la fin du chapitre « La parenthèse rhodésienne » (SM, p. 179 sq.) est tout sauf anecdotique : «Quel abîme pourtant séparait Craig de Tshikapa ou de Mama Malkia !» : en d'autres mots, ce n'est pas de ce futurlà, de ségrégation, que Harry veut pour l'Afrique : s'unir à Mama Malkia, même s'il ne s'agit pas d'un couple traditionnel, ouvre une tout autre perspective : mettre au monde cet homme nouveau qu'est Kitoko Léo.

13 Cf. Halen (P.), « La première revue Jeune-Afrique, ou les ambivalences d'un projet culturel néo-colonial au Congo belge (1947-1960) », in : ViGH (A.), éd., L'Identité culturelle dans les littératures de langue française. Paris : ACCT ; Pécs : Presses de l'Université de Pécs, 1989, 355 p. ; p.203-216. 
Songer à l'Amérique, on le sait, peut aussi nous rapprocher de l'Italie où ce rêve a inpiré nombre de départs au $20^{\mathrm{e}}$ siècle. Ce rêve peut donc bien hanter aussi les Italiens d'Élisabethville/Lubumbashi, qui, pour avoir déjà quitté le sol natal une première fois, ne rêvent pas tous d'y revenir, et l'on comprend que cela puisse être en particulier le cas des ex-Rhodiotes dont beaucoup préfèreront la Belgique, ou d'autres pays encore, lorsqu'ils seront à nouveau contraints de vider les lieux. C'est d'ailleurs le cas de la famille d'Albert Russo $^{14}$. Mais on n'en finit pas si facilement avec l'Italie, dont la présence dans Sang mêlé est d'autant plus significative qu'elle n'est que fort peu justifiée par la fiction.

L'Italie apparaît d'abord avec le personnage d'Ishaya Ben-Aharon, alias «Macaroni timide» $(S M$, p. 60), jeune condisciple et amical compagnon de Léo Kitoko, victime comme lui, à l'école, d'une exclusion de la part des autres garçons. Au cours d'une conversation entre les deux amis, Ishaya explique son origine rhodiote en accablant les « nazis [qui] avaient déporté ses grandsparents »; comme le narrateur lui demande si les Italiens «n'étaient pas aussi [leurs] ennemis », Ishaya a cette réponse stéréotypée : «les Italiens sont de braves gens, la guerre ne les intéressait pas beaucoup » $(S M$, p. 66). Comme on le sait, cette expression très répandue : Italiani brava gente, a été discutée ${ }^{15}$, mais ce n'est pas le cas ici.

Grâce à Ishaya, l'Italie revient un peu plus loin au cours d'une autre conversation entre les garçons. Ceux-ci passent devant ce qui était alors le " Musée de la Vie Indigène », que Léo Kitoko avait déjà visité lorsqu'il était à l'école primaire (une école Montessori, soit dit en passant). Ishaya raconte alors que l'immeuble avait été transformé en prison au moment de la déclaration de guerre :

Tous les Italiens d'Élisabethville y avaient été rassemblés et séquestrés en tant que prisonniers de guerre. Leurs familles leur rendaient visite chaque jour avec des paniers pleins de bons petits plats mijotés, de boissons, de fruits et de pâtisseries. Prenant cela plutôt à la rigolade, les reclus passaient leur temps à jouer aux cartes et à s'inventer d'autres diverstissements, sous l'œil complice du gardien. Ils avaient même la permission de recevoir leurs amis pour fêter leur anniversaire. Tu te figures un peu le tableau, au milieu des masques baluba? s'esclaffa Ishaya. Mon père et les autres Juifs italiens réussirent finalement à convaincre les Autorités belges de les laisser rentrer chez eux, plaidant : «Les nazis sont nos ennemis et vous nous considérez comme vos adversaires. Vous voyez bien que c'est ridicule ! ». Grâce à leur insistance, tous les Italiens furent libérés, y compris les Chrétiens. Il n'y a qu'au cinéma qu'on voit des bouffonneries pareilles. Mon père dit que les Belges ne savaient plus quoi faire pour leur rendre la vie agréable. Si toutes les prisons étaient comme cela! (SM, p. 80-81).

On peut se demander si le personnage d'Ishaya n'a pas été créé surtout pour permettre à l'auteur d'évoquer des souvenirs personnels, familiaux et « com-

14 L'auteur a gardé la nationalité belge, mais il n'a jamais vécu lui-même en Belgique où une partie de sa famille s'est néanmoins installée.

15 Voir notamment: Del Boca (Angelo), Italiani, brava gente? : un mito dura a morire Vicenza : Ed. Neri Pozza, coll. I Colibri, 2005, 318 p. 
munautaires » italiens. Le fait est que ce passage ressemble fort à un écho de la mémoire familiale de l'auteur. Un écho sans aucun doute héroïsant pour la communauté juive, qui aurait ainsi sauvé les autres Italiens, quand bien même l'Italie ne les avait guère protégés autrefois, ni de la misère ni de la menace qui pesait sur leur vie.

La présence de l'Italie ne s'arrête pas là : le nom du premier associé de Harry Wilson est « Denola », ce qui ne sonne pas particulièrement belge, mais on n'en sait pas plus. Un Docteur « Marini avec son accent italien » (SM, p. 80) intervient plusieurs fois comme médecin de famille. Plus significatif encore : Harry et son fils, cherchant un havre de paix pour se dire des choses importantes, vont se promener dans les parterres de fleurs de la «ferme Spandre », un peu à l'extérieur de la ville, qui était tenue par des colons italiens ${ }^{16}$.

L'Italie et les Italiens (dont certains, les ex-Rhodiotes, parlent espagnol) occupent donc une place très importante dans la représentation qu'Albert Russo donne de la ville où, par ailleurs, les personnages congolais sont nombreux, et quant à eux toujours en lien avec l'intérieur du pays. Les Belges y apparaissent finalement beaucoup moins, sinon sous les aspects opposés d'un missionnaire enseignant du type « dur mais juste », qui semble incarner le passé colonial (il s'appelle Kerkhof, c'est-à-dire « cimetière »), et du blond Commissaire Éric Van Pool, un jeune homme présenté comme faible et peu à sa place, qui pourrait être une image des autorités métropolitaines de l'aprèsguerre, dépassées par les réalités ${ }^{17}$. À part cela, on trouve un couple d'anglophones («Mr. et Mrs Moore », du consulat britannique - SM, p. 46), et surtout le pâtissier Giorgos dont nous avons déjà parlé, l'homme des gâteries : «le propriétaire du salon de thé où se dégustaient les meilleures pâtisseries et crèmes glacées d'Élisabethville » (SM, p. 46 ; voir aussi p. 200).

En somme, une socialité essentiellement plurielle, dont la diversité est comme garantie et illustrée par les Italiens, surtout les ex-rhodiotes. Le modèle colonial est présenté comme dépassé, le modèle rhodésien de l'« Africain blanc » constitue une séduction aussitôt délaissée : il ne reste qu'à conjuguer les deux destinées de Wilson et de Mama Malkia. Le premier, Américain en rupture d'origine, admire le pragmatisme et la solidité d'Ishayah, qui initie Léo à une double mémoire italienne et juive. La seconde, héritière d'une dynastie de chefs africains, garde un lien avec son village, où elle se réjouit d'ailleurs d'avoir laissé grandir une fille issue de son premier mariage. Le

$16 S M$, p. 219-220. Il s'agit de la ferme des parents de Mario Spandre, avocat bien connu notamment pour son activité au Katanga à l'époque de la Sécession, et plus tard à Kinshasa comme Conseiller de Moïse Tshombé premier Ministre ; il plaidera ensuite au Barreau de Bruxelles et, entre autres, témoignera devant la « Commission Lumumba ».

17 Un troisième Belge est Piet, un garçon du même âge que Léo, fils de fermiers flamands qui ont choisi de le scolariser dans une école congolaise en dehors de la ville, donc en swahili ; voir, dans ce volume, la contribution d'Edoardo Quaretta à propos des écoles salésiennes. L'aisance de Piet en milieu congolais est enviée par Léo, qui est instruit en français. 
résultat de cette conjugaison est une socialité nouvelle : en prenant en charge les destinées de Kitoko Léo qui, en tant que bâtard orphelin, aurait pu représenter les échecs et les impasses du régime colonial, Harry Wilson et Mama Malkia lui ouvrent en quelque sorte un avenir à la fois moderne et métis. C'est qu'il est pour eux «l'homme de demain » $(S M$, p. 144), tout en portant le nom d'un ancêtre africain fameux, le grand-père de Mama Malkia ${ }^{18}$.

\section{Un contenu symbolique... pour conclure}

Exercice de figuration, le roman permet la symbolisation : non pas une sorte d'allégorie abstraite, mais un narratif à haute charge symbolique. Sang mêlé figure ainsi la rencontre des cultures non comme un «choc de civilisations », mais, sans s'aveugler sur la violence toujours possible, sous le signe d'un métissage qui recommande d'être « le produit non pas d'une culture mais de plusieurs cultures, multiples facettes d'un même diamant $»^{19}$.

Le discours d'Albert Russo est clair: la neutralisation des différences installe une dynamique d'apaisement et condamne la culture des préjugés. Au lieu d'être l'objet du regard discriminatoire, l'Autre doit plutôt être le miroir par lequel nous nous réalisons dans une nouvelle identité. C'est la meilleure manière de «mettre à profit la diversité culturelle croissante de nos sociétés, la dynamique inhérente à chaque culture, et [de] créer des synergies pour inventer de nouvelles façons de vivre ensemble et d'organiser la communauté ${ }^{20}$. Même profession de foi chez Wilson :

les gens se prétendent modernes, alors qu'au fond d'eux-mêmes, ils sont pathétiquement rétrogrades. Ils voient le métis, le juif, l'hindou et ils ne perçoivent pas l'être humain. C'est dans le brassage des cultures, dans les mélanges de races, que repose notre avenir (SM, p. 144).

Une telle position dans le discours contemporain semble bien entendu indissociable du modèle que représente la collectivité italienne de ces Juifs rhodiotes devenus Katangais, et se situant ainsi au "carrefour du monde », comme d'aucuns se voyaient déjà, sur place, dans les années 1950. Une « glocalité » avant l'heure, peut-être, dont on notera qu'elle a été permise par la conjonction de plusieurs marginalités relatives. Dans le roman, Wilson, immigré américain, est surtout une figure de l'internationalité, une figure isomorphe, en somme, à celle des Italiens en Afrique, pas tout à fait «chez eux » dans des pays dont les métropoles n'étaient pas italiennes. Le métis Léo est, dans ce contexte, légèrement extérieur lui aussi aux groupes majoritaires,

18 Et même de deux ancêtres fameux, puisque, même si le roman n'en parle aucunement, le lecteur ne peut s'empêcher de faire le rapprochement entre son nom et celui de Léopold II. À noter qu'Harry Wilson meurt assassiné par les troupes de l'ONU alors qu'il est sorti de chez lui pour aller dans une pharmacie chercher des médicaments pour Mama Malkia : il incarne ainsi, presque en martyr, la fin du modèle « katangais ».

19 BLU (D.), «Introduction », art. cit., p. 7.

${ }^{20}$ BLU (D.), «Introduction », art. cit., p. 9. 
comme l'est Wilson par la sexualité, comme l'est Mama Malkia par son engagement dans une famille peu conforme à la tradition. Comme l'est ce français truffé de swahili, ou encore ce roman placé à l'enseigne d'un poème.

$* * *$ 